\title{
A GENERALIZATION OF THE SALEM TEST
}

\author{
DANIEL WATERMAN
}

(Communicated by J. Marshall Ash)

\begin{abstract}
The Salem test is a criterion for uniform convergence of a Fourier series on the circle group. An improved test for convergence at a point and uniform convergence on an arbitrary set is obtained, as well as a necessary and sufficient condition for the convergence of the Fourier series of a function at a Lebesgue point.
\end{abstract}

The result with which we are concerned is a test for the convergence of the Fourier series of a summable function. It provides conditions for convergence at a point and for uniform convergence on a set.

This result generalizes the Salem test for uniform convergence [3, 7]. Bary's opinion of the Salem test was that it appears "at a first glance to be hardly suitable for application" [3, pp. 305-310], but it has served as the starting point of many other investigations. For example, various notions of generalized bounded variation, such as Salem's $\Phi$-bounded variation [3] and Waterman's $\Lambda$-bounded variation $[9,10]$ arise directly from the ideas of the Salem test. Investigations into the everywhere convergence and uniform convergence of Fourier series under every change of variable $[1,2,4,5,6,8]$ also originated from these ideas.

We shall consider a summable real- or complex-valued function on the circle group $T$. For odd integers $n$, let

$$
\begin{aligned}
T_{n}(x, t)= & \frac{f(x+t / n)-f(x+(t+\pi) / n)}{1} \\
& +\frac{f(x+(t+2 \pi) / n)-f(x+(t+3 \pi) / n)}{3}+\cdots \\
& +\frac{f(x+(t+(n-1) \pi) / n)-f(x+(t+n \pi) / n)}{n}
\end{aligned}
$$

and let $Q_{n}(x, t)$ be obtained from $T_{n}(x, t)$ by substituting $-t$ and $-\pi$ for $t$ and $\pi$ respectively.

The Salem test can then be stated as follows.

Salem Test. If $f$ is continuous on $T$ and $T_{n}(x, 0)$ and $Q_{n}(x, 0)$ converge uniformly to 0 , then the Fourier series of $f$ converges uniformly.

Received by the editors January 13, 1988.

1980 Mathematics Subject Classification (1985 Revision). Primary 42A20; Secondary 42A16. 
Letting $S(f, x)$ denote the Fourier series of $f$, we can express our result as follows.

Generalized Salem Test. If for an $f$ in $L^{1}(T)$ we have

$$
\frac{1}{h} \int_{0}^{h}|f(x+t)+f(x-t)-2 f(x)| d t=o(1) \text { as } h \rightarrow 0
$$

and

$$
\int_{\pi}^{2 \pi}\left|T_{n}(x, t)+Q_{n}(x, t)\right| d t=o(1) \text { as } n \rightarrow \infty,
$$

then $S(f, x)$ converges to $f(x)$. If $(*)$ and $(* *)$ hold uniformly on a set $E$ and $f$ is bounded on $E$, then $S(f, x)$ converges uniformly to $f(x)$ on $E$. Condition (**) may be replaced by

$$
\int_{\pi}^{2 \pi}\left(T_{n}(x, t)+Q_{n}(x, t)\right) \sin t d t=o(1) \text { as } n \rightarrow \infty,
$$

and if $(*)$ holds at $x$, then $(* * *)$ is a necessary and sufficient condition that $S(f, x)$ converges to $f(x)$.

Remark 1. Conditions (*) states that $x$ is a (symmetric-) Lebesgue point of $f$. It would be of considerable interest to determine if condition $(*)$ can be replaced by the symmetric differentiability of $\int f$ at $x$, i.e., by

$$
\frac{1}{h} \int_{0}^{h}(f(x+t)+f(x-t)-2 f(x)) d t=o(1) \text { as } h \rightarrow 0 .
$$

It is elementary that either $(*)$ or $\left(*^{\prime}\right)$ uniformly on $T$ is equivalent to continuity.

Remark 2. It is easily seen that the denominators $(2 k-1), k=1,2, \ldots$, in the definitions of $T_{n}$ and $Q_{n}$ may be replaced by $k$.

Proof of the theorem. If $S_{n}(x)$ denotes the $n$th partial sum of the Fourier series of $f$ at $x$ and

$$
\phi_{x}(t)=f(x+t)+f(x-t)-2 f(x) \text {. }
$$

then

$$
S_{n}(f, x)-f(x)=o(1)+\frac{1}{\pi} \int_{0}^{\pi} \phi_{x}(t) \frac{\sin n t}{t} d t
$$

uniformly in $x$. Assuming (*) and integrating by parts,

$$
\begin{aligned}
\int_{0}^{\pi / n} \phi_{x}(t) \frac{\sin n t}{t} d t= & -n \int_{0}^{\pi / n}\left(\int_{0}^{t} \phi_{x}(u) d u\right) \frac{\cos n t}{t} d t \\
& +\int_{0}^{\pi / n}\left(\int_{0}^{t} \phi_{x}(u) d u\right) \frac{\sin n t}{t^{2}} d t .
\end{aligned}
$$

The first term is

$$
n \int_{0}^{\pi / n} o(1) d t=o(1)
$$


and the second is

$$
\int_{0}^{\pi / n} o(t) O\left(\frac{n}{t}\right) d t=o(1)
$$

Hence

$$
\int_{0}^{\pi / n} \phi_{x}(t) \frac{\sin n t}{t} d t=o(1)
$$

at every $x$ for which $(*)$ holds, and this is uniform on any set on which $(*)$ holds uniformly. We note that $\left(*^{\prime}\right)$ would suffice here.

In estimating $S_{n}(f, x)-f(x)$, there is no loss in generality in assuming that $n$ is odd. Letting $\sum^{\prime}$ denote summation over even indices,

$$
\begin{aligned}
\int_{\pi / n}^{\pi} \phi_{x}(t) & \frac{\sin n t}{t} d t=\sum_{k=0}^{n-2} \int_{\pi / n}^{2 \pi / n} \phi_{x}(t+k \pi / n) \frac{(-1)^{k} \sin n t}{t+k \pi / n} d t \\
= & \sum_{k=0}^{n-2} \int_{\pi / n}^{2 \pi / n}\left[\phi_{x}(t+k \pi / n) \frac{1}{t+k \pi / n}\right. \\
& \left.-\phi_{x}(t+(k+1) \pi / n) \frac{1}{t+(k+1) \pi / n}\right] \sin n t d t \\
= & \int_{\pi / n}^{2 \pi / n} \sum_{k=0}^{n-2}\left[\phi_{x}(t+k \pi / n)-\phi_{x}(t+(k+1) \pi / n)\right] \frac{\sin n t}{t+k \pi / n} d t \\
& +\int_{\pi / n}^{2 \pi / n} \sum_{k=0}^{n-2} \phi_{x}(t+(k+1) \pi / n)\left[\frac{1}{t+k \pi / n}-\frac{1}{t+(k+1) \pi / n}\right] \sin n t d t \\
= & \int_{\pi / n}^{2 \pi / n} I d t+\int_{\pi / n}^{2 \pi / n} J d t .
\end{aligned}
$$

Let $\Phi(t)=\Phi_{x}(t)=\int_{0}^{t}\left|\phi_{x}(u)\right| d u$. Then

$$
\begin{aligned}
\left|\int_{\pi / n}^{2 \pi / n} J d t\right| & =\mid \int_{\pi / n}^{2 \pi / n} \sum_{k=0}^{n-2} \phi_{x}(t+(k+1) \pi / n) \\
& \cdot\left(\frac{1}{t+k \pi / n}-\frac{1}{t+(k+1) \pi / n}\right) \sin n t d t \mid \\
& \leq \frac{n}{\pi} \sum_{k=0}^{n-2} \int_{\pi / n}^{2 \pi / n}\left|\phi_{x}(t+(k+1) \pi / n)\right| \frac{1}{(k+1)(k+2)} d t
\end{aligned}
$$




$$
\begin{aligned}
& \leq \frac{n}{\pi} \sum_{k=0}^{n-2}[\Phi((k+3) \pi / n)-\Phi((k+2) \pi / n)] \frac{1}{(k+1)^{2}} \\
& \leq \frac{n}{\pi} \sum_{k=0}^{n-3}[\Phi((k+3) \pi / n)-\Phi((k+2) \pi / n)] \frac{1}{(k+1)^{2}} \\
& \leq \frac{n}{\pi}\left[\sum_{k=1}^{n-3} \Phi((k+2) \pi / n)\left[\frac{1}{k^{2}}-\frac{1}{(k+1)^{2}}\right]+\Phi(\pi) /(n-2)^{2}\right] \\
& =\frac{n}{\pi} \sum_{k=1}^{N} \cdots+\frac{n}{\pi}\left[\sum_{N+1}^{n-3} \cdots+\Phi(\pi) /(n-2)^{2}\right] .
\end{aligned}
$$

The first term on the right is

$$
n\left(\sum_{k=1}^{N} o\left(\frac{k}{n}\right) \frac{1}{k^{3}}\right)=o(1) \quad \text { as } n \rightarrow \infty,
$$

assuming that $N / n \rightarrow 0$. The other term is

$$
\leq c n\left(|f(x)|+\|f\|_{1}\right)\left(1 / N^{2}+1 / n^{2}\right) \text {. }
$$

Choosing $N \approx n^{2 / 3}$ we see that

$$
\int_{\pi / n}^{2 \pi / n} J d t=o(1) \text { as } n \rightarrow \infty
$$

for every $x$ and that this holds uniformly on any set where $f(x)$ is bounded and $\Phi_{x}(h)=o(h)$ uniformly.

We observe now that

$$
\begin{array}{rl}
\int_{\pi / n}^{2 \pi / n} & I d t-\frac{1}{\pi} \int_{\pi}^{2 \pi}\left(T_{n}+Q_{n}\right) \sin u d u \\
= & \int_{\pi / n}^{2 \pi / n} \sum_{k=0}^{n-2}\left[\phi_{x}(t+k \pi / n)-\phi_{x}(t+(k+1) \pi / n)\right] \\
& \cdot\left[\frac{1}{t+k \pi / n}-\frac{1}{(k+1) \pi / n}\right] \sin n t d t \\
& -\int_{\pi / n}^{2 \pi / n}[f(x+t+(n-1) \pi / n)-f(x+t+\pi) \\
& +f(x-t-(n-1) \pi / n)-f(x-t-\pi)] \sin n t d t
\end{array}
$$

and this last term is dominated by $\omega_{1}(f, \pi / n)$, the integral modulus of continuity of $f$. Clearly the remaining term is $o(1)$ uniformly in $x$ if $f$ is continuous. Otherwise it is dominated by

$$
\begin{aligned}
& n \sum_{k=0}^{n-2}[\Phi((k+2) \pi / n)-\Phi((k+1) \pi / n)] /(k+1)^{2} \\
& \quad+n \sum_{k=0}^{n-2}[\Phi((k+3) \pi / n)-\Phi((k+2) \pi / n)] /(k+1)^{2}
\end{aligned}
$$


and these sums are estimated just as was done for $\int_{\pi / n}^{2 \pi / n} J d t$. Thus (***) is equivalent to

$$
\int_{\pi / n}^{2 \pi / n} I d t=o(1) \quad \text { as } n \rightarrow \infty
$$

if $(*)$ holds, implying that, under condition $(*)$, condition $(* * *)$ is necessary and sufficient for the convergence of $S(f, x)$ to sum $f(x)$. The estimate for the difference between $\int_{\pi / n}^{2 \pi / n} I d t$ and $(1 / \pi) \int_{\pi}^{2 \pi}\left(T_{n}+Q_{n}\right) \sin u d u$ is uniform on any set on which $f(x)$ is bounded.

\section{REFERENCES}

1. A. Baernstein, On the Fourier series of functions of bounded $\Phi$-variation, Studia Math. 42 (1972), 91-94.

2. A. Baernstein and D. Waterman, Functions whose Fourier series converge for every change of variable, Indiana Univ. Math. J. 22 (1972), 569-576.

3. N. K. Bary, A treatise on trigonometrical series, Vol. I, Macmillan, New York, 1964.

4. C. Goffman, Everywhere convergence of Fourier series, Indiana Univ. Math. J. 20 (1970), 107-113.

5. C. Goffman and D. Waterman, Functions whose Fourier series converge for every change of variable, Proc. Amer. Math. Soc. 9 (1968), 80-86.

6. C. W. Onneweer and D. Waterman, Fourier series of functions of harmonic bounded fluctuation on groups, J. Analyse Math. 27 (1974), 79-93.

7. R. Salem, Essais sur les séries trigonometriques, Actualités Sci. Indust., No. 862, Hermann, Paris, 1940.

8. D. Waterman, On convergence of Fourier series of functions of generalized bounded variation, Studia Math. 44 (1972), 107-117.

9. __ On $\Lambda$-bounded variation, Studia Math. 57 (1976), 33-45.

10. - , Fourier series of functions of $\Lambda$-bounded variation, Proc. Amer. Math. Soc. 74 (1979), 119-123.

Department of Mathematics, Syracuse University, Syracuse, New York 13244-1150 